Meta

Journal des traducteurs

Translators' Journal

\title{
Est-ce canadien ou non ? Les difficultés des lexicographes canadiens
}

\section{Johanne Blais et Sylvie Porhiel}

Volume 47, numéro 1, mars 2002

URI : https://id.erudit.org/iderudit/007993ar

DOI : https://doi.org/10.7202/007993ar

Aller au sommaire du numéro

Éditeur(s)

Les Presses de l'Université de Montréal

ISSN

0026-0452 (imprimé)

1492-1421 (numérique)

Découvrir la revue

Citer cet article

Blais, J. \& Porhiel, S. (2002). Est-ce canadien ou non ? Les difficultés des lexicographes canadiens. Meta, 47(1), 87-94. https://doi.org/10.7202/007993ar
Résumé de l'article

Notre article porte sur le traitement d'expressions utilisées dans deux communautés linguistiques francophones (le Canada et la France). La politique éditoriale du Dictionnaire bilingue canadien est d'indiquer à l'utilisateur les usages particuliers au Canada. Mais les expressions figées et les collocations se distinguent souvent de façon subtile d'une communauté à l'autre. Ainsi, on remarquera une différence morphologique et syntaxique dans les paires telles que tirer les cartes/tirer aux cartes, mettre les pieds dans le plat/se mettre les pieds dans les plats et faire le beau/faire la belle. De plus, il existe des collocations et des expressions qui, bien qu'identiques sur le plan formel, ne le sont pas dans leurs usages, comme par exemple avoir l'air fin et passer au travers. Ces expressions nous intéressent tout particulièrement, mais distinguer leurs usages propres au Canada n'est pas aisé pour les lexicographes canadiens. En effet, ces subtilités ne se décèlent souvent qu'en contexte, c'est-à-dire après l'analyse de corpus particuliers à chaque communauté. Nous montrons quelles sont les étapes obligatoires de notre démarche avant d'arriver à distinguer les usages propres et/ou communs à chaque communauté et de les indiquer à l'utilisateur.
Ce document est protégé par la loi sur le droit d'auteur. L’utilisation des services d’Érudit (y compris la reproduction) est assujettie à sa politique d'utilisation que vous pouvez consulter en ligne.

https://apropos.erudit.org/fr/usagers/politique-dutilisation/ 


\title{
ÉTUDES TERMINOLOGIQUES ET LINGUISTIQUES
}

\section{Est-ce canadien ou non? Les difficultés des lexicographes canadiens}

\author{
JOHANNE BLAIS ET SYLVIE PORHIEL \\ Université d'Ottawa, Ottawa, Canada
}

\begin{abstract}
RÉSUMÉ
Notre article porte sur le traitement d'expressions utilisées dans deux communautés linguistiques francophones (le Canada et la France). La politique éditoriale du Dictionnaire bilingue canadien est d'indiquer à l'utilisateur les usages particuliers au Canada. Mais les expressions figées et les collocations se distinguent souvent de façon subtile d'une communauté à l'autre. Ainsi, on remarquera une différence morphologique et syntaxique dans les paires telles que tirer les cartes/tirer aux cartes, mettre les pieds dans le plat/se mettre les pieds dans les plats et faire le beau/faire la belle. De plus, il existe des collocations et des expressions qui, bien qu'identiques sur le plan formel, ne le sont pas dans leurs usages, comme par exemple avoir l'air fin et passer au travers. Ces expressions nous intéressent tout particulièrement, mais distinguer leurs usages propres au Canada n'est pas aisé pour les lexicographes canadiens. En effet, ces subtilités ne se décèlent souvent qu'en contexte, c'est-à-dire après l'analyse de corpus particuliers à chaque communauté. Nous montrons quelles sont les étapes obligatoires de notre démarche avant d'arriver à distinguer les usages propres et/ou communs à chaque communauté et de les indiquer à l'utilisateur.
\end{abstract}

\begin{abstract}
This paper deals with the treatment of expressions used in two French-speaking regions, Canada and France. According to the editorial policy of the Bilingual Canadian Dictionary, all usages particular to Canada must be marked in this work. But there are often very subtle differences in fixed expressions and collocations from one region to another. For example, there are only slight morphological or synctactic differences in the following expressions: tirer les cartes/tirer aux cartes, mettre les pieds dans le plat/se mettre les pieds dans les plats et faire le beau/faire la belle. In addition, there are expressions, which are of even more interest to us, that are identical in form but not in the way they are used, expressions such as avoir l'air fin et passer au travers. It is certainly not easy for Canadian lexicographers to determine the specific Canadian usage of such expressions. The only way to do so is to study these expressions in context, i.e., to analyze a representative corpus from each region. This paper presents the stages that Canadian lexicographers must go through if they are to distinguish the usages particular to a given community and to mark these differences in a dictionary.
\end{abstract}

\section{MOTS-CLÉS/KEYWORDS}

canadianismes, dictionnaires, lexicographie, usage, marquage 


\section{Introduction}

Les expressions sont un des moyens d'expression privilégiés des locuteurs. Elles «sont le plus souvent imagées et familières: elles mettent dans le discours une couleur que les énoncés régulièrement produits n'ont pas. En même temps, elles sont fixées, traditionnelles et souvent caractéristiques d'une classe, d'un milieu, d'un état de la société»(Rey et Chantreau 1979: vii).

Les pays francophones ont en commun un grand nombre d'expressions qui sont ou ne sont pas sémantiquement équivalentes ou qui ne le sont que partiellement ${ }^{1}$. Il arrive que des usagers consultent les dictionnaires pour savoir si telle expression s'emploie en France et au Canada, ou dans une seule des communautés linguistiques, et dans quelle situation de communication. Comme les expressions peuvent varier d'une communauté francophone à une autre, une mauvaise compréhension peut engendrer des problèmes de traduction, voire de communication. C'est pourquoi dans le Dictionnaire canadien bilingue (DCB), nous guiderons les locuteurs à l'aide de la marque $\mathrm{CD}$ (canadianisme), en identifiant à quelle communauté appartient telle expression et quel(s) sens il faut lui donner ${ }^{2}$. Mais distinguer ce qui est canadien de ce qui ne l'est pas présente des difficultés, car les expressions varient à plus d'un égard et sont souvent d'un usage différent. En effet, "c'est dans cette partie de la langue qu'on observe le plus grand nombre de différences entre le québécois et le français standard» (Meney: $\mathrm{xx}$ ). On remarque ainsi de nombreuses différences morphologiques, syntaxiques et lexicales dans les paires d'expressions ci-après: allez chez le diable (CD) / aller au diable; tirer aux cartes (CD) / tirer les cartes; se mettre les pieds dans les plats (CD) / mettre les pieds dans le plat; faire la belle (CD) / faire le beau; jouer cartes sur table (CD) / mettre cartes sur table. Plusieurs de ces écarts morphosyntaxiques et lexicaux subtils ont déjà été étudiés ${ }^{3}$, c'est pourquoi nous nous attarderons plus particulièrement sur des expressions qui, bien qu'identiques sur le plan formel, ne le sont pas nécessairement sémantiquement, comme par exemple, avoir l'air fin, passer au travers, etc. Les exemples d'expressions de même forme et de sens différent sont moins fréquents et, comme nous le verrons, plus difficiles à identifier et à interpréter que les expressions qui varient du point de vue formel.

Dans cet article, nous exposerons tout d'abord les difficultés que nous rencontrons pour déterminer si une expression de même forme a le même sens ou des sens différents. Nous illustrerons ensuite notre démarche à l'aide d'exemples concrets.

\section{Nos difficultés}

La première difficulté du lexicographe canadien est de ne pas savoir d'emblée si une expression appartient au français du Canada et/ou au français de France. Tout comme l'usager, le lexicographe doute et cherche des réponses dans les dictionnaires. En effet, les écarts entre l'usage franco-canadien et l'usage français, facilement décelables pour les Français de l'Hexagone, le sont beaucoup moins pour les francophones vivant au Canada. Comme il n'existe pas encore de dictionnaire complet du français canadien, il faut donc consulter plusieurs ouvrages. C'est un long processus qui s'avère parfois frustrant, car les informations qu'ils contiennent sont souvent incomplètes, imprécises, voire erronées. Par exemple, il arrive qu'une expression soit notée propre au Canada alors qu'après vérification elle ne l'est pas. Il arrive aussi qu'une 
expression soit répertoriée mais qu'elle ne soit pas expliquée ou pas illustrée. Il faut même parfois travailler par déduction, c'est-à-dire que si l'expression n'est pas consignée dans un des grands dictionnaires français, c'est peut-être qu'elle est canadienne. En outre, pour dresser un tableau complet des sens de certaines expressions polysémiques, il faut souvent compiler les différents sens attestés dans les dictionnaires. Enfin, certaines combinaisons de mots qui forment des expressions au Canada ne sont que des combinaisons libres en France. Dans ce cas, ce n'est souvent que le contexte qui permet d'affirmer qu'il s'agit d'une expression ou d'une combinaison libre.

\section{Notre démarche}

Lors de l'analyse des expressions, nous visons une démarche qui soit la plus rigoureuse et la plus objective possible même si nous sommes conscientes qu'il existe une part inévitable d'arbitraire. C'est pourquoi chaque expression fait l'objet de vérifications dans plusieurs ouvrages dictionnairiques. Nous consultons les principaux ouvrages sur le français du Canada (Bélisle, Bergeron, Clas et Seutin 1979 et 1989, Dulong, Meney, Poirier, DQA); les principaux dictionnaires du «français standard » (GL, GR, TLF), les dictionnaires d'expressions (Rey et Chantreau, Duneton); les principaux dictionnaires bilingues (RCSS, OXHA, LAR).

Toutefois, nous ne pouvons limiter nos recherches aux seules informations consignées dans les dictionnaires. Ainsi, nous faisons une recherche systématique des expressions dans des corpus français du Canada (la Presse canadienne-française (PCF), 77 millions de mots; Leméac (LEM), 0,9 millions et de France (Ouest-France (OF), 4,9 millions; Le Monde (MOND), 17,1 millions) afin de déterminer l'usage réel de l'expression. Nous utilisons par ailleurs les ressources qui nous sont accessibles par Internet comme le corpus bilingue TransBase composé de textes parlementaires et la base de données du Trésor de la langue française. Nous faisons aussi appel à des informateurs.

Idéalement, cette démarche devrait soit confirmer les informations consignées dans les dictionnaires, soit les infirmer. Toutefois, malgré les outils dont nous disposons, certains doutes subsistent.

\section{Exemples}

\section{L'expression est-elle propre au Canada?}

Les critères qui servent à apposer la marque CD varient d'un auteur à l'autre et ne font pas nécessairement l'objet d'un consensus. En outre, plusieurs des ouvrages consultés datent et surtout la langue est en constante évolution. C'est pourquoi ce n'est pas parce qu'une expression est marquée canadienne dans des ouvrages traitant du français canadien que nous reprenons systématiquement cette marque.

Considérons, par exemple, l'expression ne pas avoir la/sa langue dans la/sa poche notée CD dans Bergeron et Dulong ${ }^{4}$ L'alternance morphosyntaxique n'étant pas la raison justifiant cette marque ${ }^{5}$, nous avons dû vérifier s'il existe des différences sémantiques entre le français international et le français canadien. Comme Dulong et Bergeron expliquent l'expression ne pas avoir la/sa langue dans la/sa poche par une 
autre expression avoir la langue bien pendue, il a fallu tout d'abord vérifier le sens d'avoir la langue bien pendue, soit, selon GR et TLF, "parler beaucoup, facilement». Toutefois, notre sentiment est que, au Canada, l'expression ne pas avoir la/sa langue dans la/sa poche s'utilise dans le sens de «ne pas avoir peur de dire ce que l'on pense» et non pas «parler facilement». Le DQA confirme notre intuition et donne la définition «ne pas craindre de dire ce que l'on pense» et "avoir de la répartie». Mais comme le DQA donne à la fois les sens canadiens et les sens français sans les distinguer, nous ne pouvions pas déduire si «dire ce que l'on pense» était propre au français du Canada. Nous avons donc comparé ce sens à ceux répertoriés dans les dictionnaires du «français standard»: GR «parler avec facilité, abondance», GL et TLF «avoir la répartie vive», Rey et Chantreau «parler avec facilité et, notamment, répliquer». On constate que tous les dictionnaires mentionnent le sens «parler avec facilité » et qu'en revanche aucun explicitement celui de «ne pas craindre de dire ce que l'on pense». En analysant l'usage réel de l'expression dans les corpus, on ne relève que le sens «ne pas craindre de dire ce que l'on pense» dans le corpus canadien; dans le corpus européen, on trouve également ce sens et quelques occurrences de «parler avec facilité». En conclusion, on aurait tort de reprendre la marque CD proposée par Dulong et Bergeron, car un des sens de ne pas avoir la/sa langue dans la/ sa poche est, selon le GR "parler avec facilité, abondance», même s'il est moins connu ici. Quant à l'autre sens de l'expression, soit «ne pas craindre de dire ce que l'on pense", nous ne le considérons pas CD, car même si les dictionnaires n'utilisent pas ces mots pour la définir, mais plutôt: «répliquer» (Rey et Chantreau), «avoir la répartie vive»(GL), «avoir la répartie prompte»(Duneton), ces nuances ne justifient pas, à notre avis, l'apposition de la marque CD.

Examinons à présent l'expression faire un tour que Bergeron, Bélisle et Dulong considèrent CD dans le sens de «rendre visite». Ces derniers illustrent cette expression ainsi: viens faire un tour avec ton mari après souper (Bélisle), venez faire un tour, ne comptez pas vos tours, on aime toujours ça vous voir (Dulong). Il est important de remarquer que, dans ces exemples, il faut sous-entendre chez qn. C'est donc faire un tour suivi de la préposition chez qui nous a posé problème. Dans GL et GR, on trouve l'expression avec le sens de «sortie» ou "déplacement» mais non suivie de chez. Le TLF donne faire un tour chez qn pour illustrer encore une fois le sens de "déplacement, promenade». Le DQA, étonnamment, ne donne pas le sens de «visite» et explique l'expression ainsi: "une petite sortie». Le dictionnaire bilingue OXHA donne l'exemple je vais faire un tour chez des amis ${ }^{6}$ qui illustre bien le sens mentionné dans les ouvrages traitant du français du Canada. Avant de pouvoir nous prononcer définitivement sur l'usage canadien ou non de l'expression, nous avons cherché des attestations de l'usage réel de l'expression. Nous avons trouvé plusieurs occurrences dans le corpus canadien illustrant le sens de "visite» suivies ou non de chez. Nous n'avons trouvé aucun exemple dans notre corpus européen, mais la base de données du Trésor de la langue française nous a fourni plusieurs occurrences de faire un tour chez qn dans le sens de «rendre visite». Ce sont ces exemples qui nous ont finalement convaincues de ne pas étiqueter l'expression faire un tour chez qn $\mathrm{CD}^{7}$.

Nous avons dû procéder à des vérifications similaires pour les expressions lever le coude, piquer un somme $e^{8}$, se ressembler comme deux gouttes d'eau qui sont aussi notées $\mathrm{CD}$ dans certains dictionnaires, mais qui, après analyse, s'avèrent ne pas l'être. 


\section{L'expression a-t-elle un ou des sens différent(s)?}

Les expressions que nous avons examinées jusqu'à présent ont le même sens au Canada et en France. Mais on trouve aussi des expressions qui présentent un autre niveau de difficulté parce que, bien qu'elles soient utilisées dans les deux communautés, elles le sont parfois de la même façon, mais aussi parfois de façon différente.

Avoir l'air fin est consignée dans le GL, TLF, Rey et Chantreau et Duneton avec le sens d' «avoir l'air idiot, ridicule». Le GLE donne «avoir l'air ridicule à la suite d'une déception, d'une erreur, etc.» (iron.). Les dictionnaires canadiens donnent les sens suivants: "être distingué dans sa tenue, dans ses manières; bien paraître » (Poirier); "avoir l'air gentil, sympathique» (Meney); "avoir l'air distingué, élégant, être joli, beau», "être en mauvaise posture, mal pris» (DQA). Comme le DQA est le seul dictionnaire à donner à avoir l'air fin le sens d' «être en difficulté», nous avons dû nous poser la question: quand on est en difficulté, a-t-on nécessairement l'air idiot? Sans doute, par ironie, comme le sous-entend l'exemple du DQA: Tu as l'air fin avec ton auto prise dans le banc de neige. L'expression avoir l'air fin aurait donc trois sens: deux appartenant au français du Canada et un appartenant au «français standard». Pour arriver à cette conclusion et pour établir une liste complète des sens - celui partagé par la communauté francophone et ceux particuliers au français du Canada -, nous avons dû consulter plusieurs ouvrages, aucun dictionnaire ne répertoriant tous les sens. Dans le corpus européen, on ne trouve aucune occurrence de l'expression dans $\mathrm{OF}$ et seulement 2 dans MOND dans le sens «avoir l'air ridicule». Dans le corpus canadien PCF, 1 occurrence confirme le sens «être distingué dans sa tenue », et aucune occurrence ne confirme «avoir l'air gentil»; 8 occurrences illustrent le sens « avoir l'air ridicule».

Le traitement de l'expression passer à/au travers ${ }^{9}$ dans les dictionnaires canadiens est étonnant. Le DQA en illustre ainsi le sens: Il est passé au travers des pires difficultés, suivi de l'explication, «les a évitées, y a échappé». Selon nous, cet exemple prête à confusion car, pour la plupart des Canadiens, passer au travers de difficultés signifie les surmonter et non pas y échapper! Le PLUS et le CEC donnent également à passer au travers le sens "d'échapper à quelque chose». Seutin et Clas ne donnent qu'un sens à l'expression, soit celui de «terminer, achever» et l'illustrent par: Marguerite (...) suivait la ligne jusqu'au bout et passait au travers du livre. Le GR note l'expression familière et la définit ainsi: "échapper à un danger, à une punition ». Le GL définit l'expression par «être dispensé fortuitement ou se faire dispenser habilement de quelque chose d'ennuyeux, de pénible» et en construction absolue "échapper à quelque chose »; il nous éclaire davantage grâce aux exemples fournis: passer au travers d'une corvée. Passer à travers tous les contrôles. Tous ses camarades ont été punis, mais lui est passé à travers. L'expression se trouve dans les principaux dictionnaires bilingues mais, comme on pouvait s'y attendre, aucun ne note les sens canadiens. Tous proposent to escape comme équivalent général, et illustrent ainsi l'expression: passer au travers d'une corvée to get out of doing a chore; tout le monde a eu la grippe mais je suis passé au travers everyone had the flu but I managed to avoid ou escape it (RCSS); il y a eu des licenciements, heureusement il est passé au travers there have been redundancies, fortunately his job wasn't affected (OXHA); passer au travers des dangers to escape danger (LAR).

Il y a plus de 500 occurrences de l'expression passer à/au travers dans le corpus canadien. Leur analyse permet de constater une disparité entre le sens donné par les 
dictionnaires consultés et l'usage réel de l'expression. En effet, passer au travers n'a jamais le sens d' «échapper» dans les contextes étudiés, mais plutôt ceux de 1) «s'en sortir, surmonter» et 2) «lire d'un bout à l'autre». Il n'y a que 13 occurrences de passer à/au travers au sens figuré dans le corpus européen. La plupart des contextes confirment le sens "d'échapper à quelque chose», comme dans passer au travers des contrôles, de sanctions, la loi. Dans deux exemples, passer au travers a le sens de «subir» comme dans Ces jeunes officiers vont devoir passer à travers une série d'épreuves, sur le terrain et en écoles (MOND) et celui de "surmonter» comme dans La région ne passe pas au travers de la crise économique actuelle même si elle résiste mieux que l'ensemble de l'Hexagone (OF). Ces sens ne sont pas explicitement répertoriés dans les dictionnaires $\mathrm{du}$ «français standard». Seul le GLE donne un exemple similaire pour illustrer le sens «en traversant qqch de part en part»: Il a couru de grands dangers, mais il est passé au travers. Ici l'expression n'a pas le sens «d'échapper» mais celui de «traverser» au sens figuré, c'est-à-dire «surmonter».

Lionel Meney est le seul à donner à passer à travers les sens suivants: "endurer, subir, supporter, venir à bout de, surmonter, se sortir de, se tirer d'affaire, aller jusqu'au bout de». Il donne de nombreux exemples dont passer à travers un divorce, des difficultés, une crise que l'on retrouve dans le corpus canadien. Il donne aussi passer au travers au sens de "aller jusqu'au bout, lire d'un bout à l'autre». En ce qui concerne les sens de «subir, surmonter", un doute subsiste encore. En effet, au Canada et en France, il semble que l'expression, suivie de certains mots comme crise, difficultés, épreuves, pourrait, d'après le corpus, avoir le même sens. Meney mentionne que l'anglais dit to go through et suggère ainsi indirectement que passer au travers est un anglicisme. Nous sommes d'accord avec lui pour ce qui est du sens de "terminer, achever», mais pas forcément pour celui de «surmonter». Notons tout d'abord que nous avons trouvé dans la base de données du Trésor de la langue française une attestation, en vieux français, de passer au travers de difficultez. Ensuite, passer au travers n'est répertorié comme anglicisme ni dans le Colpron ni dans le Multi; l'OLF ne le mentionne pas non plus. On donne pourtant passer une loi, des règlements, passer des remarques, passer le chapeau, etc.

L'expression passer au travers existe en «français standard», mais elle a des sens différents et probablement aussi une fréquence d'emploi différente. Comme elle est très courante au Canada, mais n'est jamais employée au sens "d'échapper"10, il est important que le DCB reflète cet usage et propose les équivalents qui correspondent aux sens canadiens. Utiliser le seul équivalent to escape proposé par les dictionnaires bilingues existants mènerait à des contre-sens dans des phrases comme, passer au travers d'une grippe, d'une épreuve, d'une difficulté, où passer au travers a au Canada le sens de «s'en sortir» et non pas celui "d'y échapper».

Tout comme pour passer au travers, nous avons dû pour les expressions partir pour la gloire, perdre la carte, tirer la patte déterminer quels étaient les sens communs aux deux communautés et quels étaient ceux spécifiquement canadiens.

\section{Expression ou combinaison libre ${ }^{11}$ ?}

En tant que locuteurs, nous savons que certaines combinaisons de mots constituent des expressions. Ainsi, au Canada, la suite de mots passer tout droit veut dire: 1) «ne pas se réveiller à temps» (Meney), 2) «dépasser par inadvertance son point de desti- 
nation» (Dulong). Le DQA, quant à lui, ne donne qu'un sens « dépasser son point de destination par inadvertance» avec les exemples: Je n'ai pas reconnu la maison et je suis passé tout droit. Passer tout droit au feu rouge, à l'arrêt, ne pas s'arrêter ${ }^{12}$. Cet usage canadien constaté, nous avons dû vérifier si l'expression était également répertoriée dans les dictionnaires du «français standard», ce qui n'est pas le cas. Dans le corpus canadien, on retrouve les sens mentionnés dans les dictionnaires; en revanche, dans le corpus européen, l'occurrence de passer tout droit n'illustre que le sens concret de "passer directement»: les graines passent tout droit dans le système digestif. Cette expression sera donc étiquetée CD.

Il n'y a rien là (il n'y a pas de problème, c'est simple comme bonjour), lever le nez (sur) (faire le difficile, traiter de haut), ne lâche pas! (tiens bon!), ne pas laisser / donner sa place (ne pas avoir son pareil), passer sous/en dessous de la table (être privé de repas; sauter un repas) ${ }^{13}$, sont d'autres exemples de suites de mots qui constituent des expressions au Canada, mais ne sont que des combinaisons libres possibles en France.

\section{Conclusion}

«Démêler l'écheveau des francophonismes, c'est-à-dire des unités lexicales communes à tous les francophones, et des particularismes n'est pas une sinécure» (Boulanger: 181). C'est pourtant le défi que tentent de relever les lexicographes du DCB chaque fois qu'ils analysent une expression et qu'ils doivent décider si elle fait ou non partie de l'usage canadien et qu'ils sont aux prises avec les disparités des dictionnaires, les limites des corpus et leur propre jugement. Malgré les difficultés qu'ils rencontrent et que nous venons d'exposer, nous sommes tout de même convaincues que l'usager en sort gagnant, car la marque CD concourt à satisfaire ses attentes et ses besoins, et à lui assurer une plus grande sécurité linguistique.

\section{NOTES}

1. G. Klein et B. Lamiroy proposent de répartir les expressions dans quatre catégories : a) même forme, même sens; b) même forme, sens différent; c) forme différente, même sens; d) forme différente, sens différent.

2. Le marquage de certains mots et expressions dans le DCB, que ce soit du français vers l'anglais ou de l'anglais vers le français, relève d'une politique éditoriale. Il correspond aussi aux attentes des utilisateurs canadiens (Cajolet-Laganière 1998).

3. Voir les travaux réalisés dans le cadre du projet FBQS, soit $\mathrm{F}$ (rançais), B(elgique), Q(uébec), S(uisse).

4. Il faut signaler que l'expression a été ajoutée dans l'édition de 1999 du Dictionnaire des canadianismes.

5. On remarque également l'alternance $l a / s a$ dans les dictionnaires du «français standard».

6. Notons que cet exemple illustre tour au sens de «déplacement» et non pas «visite».

7. Il est par ailleurs intéressant de noter l'usage fréquent de l'expression faire un tour chez qn sur Internet dans le sens de "visiter la page ouèbe de qn».

8. Dulong, dans son édition de 1989, note l'expression piquer un somme CD et ne la fait plus apparaître dans l'édition revue et corrigée de 1999.

9. Comme Rey et Chantreau, nous considérons que à/au travers de sont synonymes. C'est l'emploi figuré qui nous a posé problème et non pas son sens concret «de part en part», comme dans le ballon est passé au travers de la fenêtre.

10. Ce sens de passer au travers n'est pas connu ici, au Canada. Même des traducteurs chevronnés à qui on a posé la question nous ont avoué qu'ils ne savaient pas que passer au travers pouvait vouloir dire échapper à quelque chose. À la question, que veut dire pour vous passer au travers?, ils ont tous répondu «surmonter, s'en sortir». 
11. Nous considérons seulement le cas où une expression dans le français du Canada correspond à une combinaison libre dans le «français standard». Néanmoins, l'inverse est également vrai et une expression en «français standard» peut correspondre à une combinaison libre en français du Canada, comme par exemple avoir le coup qui correspond à avoir le tour (CD).

12. Cet exemple correspond en fait à un troisième sens de l'expression passer tout droit, soit celui de «ne pas s'arrêter».

13. Les sens canadiens de ces expressions sont tirés de Meney.

\section{RÉFÉRENCES}

BéLisLe, L.-A. (1974): Dictionnaire de la langue française au Canada, Québec et Sondec, Montréal. Bergeron, L. (1980): Dictionnaire de la langue québécoise, VLB, Montréal.

Boulanger, J.-C. (1998) : «Le pacte normatif du français québécois: réflexions sur les marques lexicographiques diatopiques», in Louis Mercier et Claude Verreault: 171-187.

CEC (Dictionnaire CEC Jeunesse) 1986, Nouvelle édition revue et augmentée, CEC, Montréal.

Clas, A. et E. Seutin (1979): Richesses et particularités de la langue écrite au Québec, Département de linguistique et philologie, Université de Montréal, Montréal, 8 vol.

Clas, A. et E. Seutin (1989): J'parle en tarmes: dictionnaire de locutions et d'expressions figurées au Québec, Sodilis, Montréal.

Colpron, G. (1994): Le nouveau dictionnaire des anglicismes, Beauchemin, Laval.

Cajolet-Laganière, H. (1998) : «Attentes et besoins du public québécois en matière de dictionnaires de langue», in Louis Mercier et Claude Verreault: 61-70.

DQA (Dictionnaire québécois d'aujourd'hui) 1993, Dicorobert, Québec.

Dugas, A. et B. Soucy (1991): Dictionnaire pratique des expressions québécoises, Éditions Logiques, Montréal.

Dulong, G. (1999): Dictionnaire des canadianismes, nouv. éd. rev. et augm., Septentrion, Sillery.

Duneton, C. (1990): Le Bouquet des expressions imagées: encyclopédie thématique des locutions figurées de la langue française, Le Seuil, Paris.

GL (Grand Larousse de la langue française; 7 vol.) 1986, Larousse, Paris.

GLE (Grand Larousse Encyclopédique; 10 vol.) 1964, Larousse, Paris.

GR (Grand Robert) 1987, Le Robert, Paris.

KLeIN, J.-R. et B. LAmiroy (1995): «Expressions figées de Belgique», Linguisticae Investigationes supplementa $17: 37-52$.

LABELLE, J. (1995): «Le traitement automatique des variantes linguistiques en français: l'exemple des "concrets" ", Linguisticae Investigationes 19, 1: 137-152.

LAR (Dictionnaire français anglais) 1993, Larousse, Paris.

Meney, L. (1999) : Dictionnaire québécois français - pour mieux se comprendre entre francophones, Guérin, Montréal.

Mercier, L. et C. Verreault (1998): Les marques lexicographiques en contexte québécois, Actes de la table ronde tenue à Montréal les 3 et 4 novembre 1994, Gouvernement du Québec, Québec.

MULTI (Dictionnaire des difficultés de la langue française), 1992, nouvelle édition mise à jour et enrichie, Québec/Amérique, Montréal.

OXHA (Oxford-Hachette French Dictionary) 1994, OUP, Oxford.

PLUS (Dictionnaire du Français Plus à l'usage des francophones d'Amérique du Nord) 1988, CEC, Montréal.

PoIrIer, C. (dir.) 1998: Dictionnaire historique du français québécois, Les Presses de l'Université Laval, Sainte-Foy.

RCSS (Roberts \& Collins Super Senior, Dictionnaire français-anglais anglais-français) 1995, HarperCollins Publishers-Le Robert, Glasgow, Paris.

Rey, A. et S. Chantreau (1985): Dictionnaire des expressions et locutions, Robert, Paris.

TLF (Trésor de la Langue Française) 1971, CNRS, Paris. 